\title{
Surfaces
}

\section{LA CULTURE EN TANT QU'ACTION POLITIQUE}

\section{Karlheinz Barck}

Volume 2, 1992

ACTES DU COLLOQUE « REPENSER LA CULTURE » ACTS OF THE CONFERENCE "RETHINKING CULTURE"

URI : https://id.erudit.org/iderudit/1065220ar

DOI : https://doi.org/10.7202/1065220ar

Aller au sommaire du numéro

\section{Éditeur(s)}

Les Presses de l’Université de Montréal

ISSN

1188-2492 (imprimé) 1200-5320 (numérique)

Découvrir la revue

Citer ce document

Barck, K. (1992). LA CULTURE EN TANT QU'ACTION POLITIQUE. Surfaces, 2. https://doi.org/10.7202/1065220ar
Résumé de l'article

Notes sur la problématique du concept de culture dans la pensée allemande contemporaine. La nécessité de repenser la culture comme action politique sans référence à Rousseau. d'utilisation que vous pouvez consulter en ligne. 


\section{ACTES DU COLLOQUE}

"REPENSER LA CULTURE"

\section{LA CULTURE EN TANT QU'ACTION POLITIQUE}

$\underline{\text { Karlheinz Barck }}$

Cette conférence a été donnée au colloque Repenser la culture qui s'est tenu à l'Université de Montréal, les 3, 4 et 5 avril 1992. Les organisateurs tiennent à remercier, pour leur soutien financier:

le Conseil de Recherche en Sciences Humaines du Canada la Faculté des Arts et des Sciences, Université de Montréal

le Vice-rectorat à l'enseignement et à la recherche, Université de Montréal

le Département de Littérature comparée, Université de Montréal Alitalia

RÉSUMÉ

Notes sur la problématique du concept de culture dans la pensée allemande contemporaine. La nécessité de repenser la culture comme action politique sans référence à Rousseau.

\section{ABSTRACT}


Notes on the problem of the concept of culture as addressed in contemporary German thought. The necessity of rethinking culture without reference to Rousseau, as a form of political action.

O. Quelle est la visée (ou le compromis) du titre qu'on a donné à cette table ronde? Réfléchissant sur sa ou ses portée(s), je dirais qu'il y a au moins deux façons de le comprendre: la culture comme champ et/ou médium d'action politique d'un côté, et la culture comme objet d'action politique de l'autre côté. Si l'on accepte le point de vue selon lequel des concepts politiques sont toujours traversés par leurs opposés, par une tension polémique et critique, sans lesquels ils ne seraient que des abstractions vides, on peut se poser d'autres questions. La notion de "culture" (aujourd'hui) a-t-elle besoin expressément d'être mise en relation avec un espace d'action politique? Nous croyons-nous obligés de rappeler une évidence (Selbstverständlichkeit Selbstverständlichkeit ) qui aurait été oubliée ou perdue à savoir que la culture comporte toujours une, voire plusieurs dimensions politiques? $\mathrm{Ou}$ s'agirait-il plutôt d'une indication restreinte, encadrée et particularisante, de la détermination spéciale d'un champ politique et qui impliquerait l'existence d'autres champs de la culture qui ne seraient pas politiques? Dans un tel cas, cela reviendrait à dire que la culture peut être aussi action politique. Il ne s'agit pas là de questions purement rhétoriques ou naïves. Récemment, Jurgen Habermas a constaté une "nouvelle intimité entre politique et culture" qui aurait écarté une fois encore (et peut-être définitivement) toute forme d'esthétisme culturel. Selon Habermas, cette intimité consisterait en une "politique symbolique" qui aurait tendance à décentraliser des possibilités de contestation (ou de résistance:

Dezentralisierung der Widerspruchsmöglichkeiten ) dans nos sociétés modernes de culture (Kulturgesellschaft ). Cette tendance fait ainsi ressortir le caractère tout à fait ambivalent de cette intimité. Les questions soulevées n'ont donc pas de réponses rapides. On ne peut les résoudre ni par queque préjugé sorti comme un lapin du chapeau, ni par une profession de foi à bon marché. J'aimerais contourner l'enjeu qu'elles impliquent en soumettant quelques thèses (ou hypothèses).

1. Il y a des sociologues de la culture qui tirent de leur analyse de la soidisant "société de loisirs" (Freizeitgesellschaft, leisure society ) au sein des soi-disant sociétés post-industrielles, la conclusion que le caractère du travail a été transformé de façon si profonde que ce n'est plus le travail qui est le médium dominant de la socialisation, mais la culture qui l'a remplacé dans ce sens et qui est devenue le médium décisif des modes et formes de socialisation. Certains voient dans cette tendance nouvelle la compensation d'un manque (manque d'expériences, d'événements, de Erlebnisse ) qui pourrait se transformer en créativité, en recherches d'alternatives de vie sociale. Se référant à la situation en RFA, l'éditeur de la prestigieuse revue "Esthetik und Kommunikation " observait en 1987, par exemple: 
En République Fédérale d'Allemagne nous nous trouvons en face d'un boom culturel sans précédent. Jamais il n'y a eu une telle affluence à des événements culturels. À tous les niveaux de la société et dans toutes les générations se fait sentir un désir d'événements et d'expériences qui puissent créer l'illusion (et affirmer l'ambition) de sortir de la vie quotidienne.

Ce que Victor Turner et Richard Schechner aux États-Unis ont appelé le surgissement d'une subjunctive culture fait état de désirs analogues, bien qu'en y réagissant d'une façon plus critique et politique à la fois. Il est significatif que le travail de la culture en dehors des mainstreams se définit comme politique dans le sens de "Working Hypotheses for a PostRevolutionary Society" comme l'envisage, par exemple, Michael Ryan dans son livre Politics and Culture:

The post-structuralist theory of culture breaks down the boundary between the traditional concept of culture, conceived roughly as the arts, the media, and symbolic social relations, and the supposedly extra-cultural realm of materiality, economics, social reality, and so forth. (Ryan, Politics and Culture: Working Hypothesis for a postrevolutionary society [Baltimore: Johns Hopkins University Press, 1989], p.13)

Thus, rather than being nothing more than an imaginary resolution, subcultural styles might also be seen as representing a potential and as prefiguring a possibility. The question posed by the post-culturalist theory of culture would go something like this: is culture a secondary representation or embodiment of a group life substance that is assumed to be prior and that is expressed through culture, or does culture, defined as shape, form, representation, embodiment, and objectification play a more primary constitutive role in the making of group life. Prior to the cultural representation, is there a pre-representational life that delegates itself into representation, leaving its substantive presence temporarily, or is that life itself structural by representations? When one analyzes culture in relation to society, does one move from culture to life substance or does one move from culture to culture? Can group life even be said to be at all possible prior to cultural embodiment or form? (Ryan, p. 21)

To derive an analogy from Marx, just as capitalism at its furthest reaches is also the first indication of the possibility of communism, so also, one could say, capitalist culture at its furthest reaches (where culture or simulation subsumes reality entirely) becomes the instrument for fabricating a postcapitalist world... (Ryan, p. 83)

Dans ce sens, le(s) concept(s) de subjunctive culture est (sont) une nouvelle mise à jour de ce qui a été depuis l'Aufklärung la fonction d'anticipation des philosophes et de la culture artistique et intellectuelle, interprétée par Marx 
comme a-symétrie temporelle de la culture d'une époque vis-à-vis de la structure économique et sociale (ou encore comme Vorschein par Ernst Bloch, le philosophe du "Prinzip Hoffnung ").

Ce qui pourtant fait problème, aujourd'hui, c'est que cette vision optimiste de l'espoir était fondée sur l'idée (ou sur la croyance) que la forteresse Europe tiendrait son rôle d'avant-garde à perpétuité: une vision qui comptait avec le consentement du Tiers Monde qui a été victime de la modernité. Ce conflit (ou cette contradiction) se double par un nouveau décalage entre le niveau du progrès économique et technique, et celui de la conscience et de la réflexion (ou plutôt de la prise de conscience de ces conflits).

Je vais illustrer cette observation par un exemple. Il y a quelques mois on a pu voir à la TV allemande un talk-show avec un auteur connu de la "littérature de masse", Konsalik, violemment attaqué par la modératrice à cause de "la nullité politique et artistique" de ses romans. Ce monsieur ne comprenait pas du tout ces critiques et, sans s'échauffer outre mesure, fit valoir le fait que ses livres ont eu un tirage total de 77 millions d'exemplaires, tirage bien supérieur à celui de 65 Prix Nobel de littérature mis ensemble.

Heiner Muller (toujours président de l'Académie des Arts à Berlin-Est) a commenté ce phénomène révélé par Konsalik en disant que les "sociétés de culture" sont arrivées à un état d'entropie de leur réflexion critique, un genre de stagnation de la conscience et d'abaissement de tout niveau culturel. L'envers du développement éblouissant du potentiel économique et technique dans les pays développés serait une véritable paupérisation intellectuelle: "À partir d'un certain niveau de sécurité économique on n'a plus envie de penser. Penser comme une activité et comme une joie pour vaincre des résistances (Überwindung von Widerständen ) est considéré comme trop fatiguant. On préfère aller au cinéma ou bien on se plaît dans le temps-vide que crée la TV. Ce sont là les facteurs qui ont fait échouer le projet de l'Aufklärung. À partir d'un certain moment, l'évolution de l'économie et l'évolution de la conscience vont dans des directions opposées à l'extrême." Puis il a proposé d'établir un programme sur ordinateur qui mettrait en relation le sous-développement économique du Tiers Monde et la paupérisation intellectuelle du Premier Monde.

Pour reprendre l'observation sociologique citée au début en ce qui concerne la culture devenue champ et facteur prépondérants de socialisation, la question doit être posée (et discutée): de quelle culture, de quel lieu et de quelle socialisation s'agit-il?

2. Avec ma deuxième thèse, je vais essayer d'envisager une perspective possible pour répondre à ces questions. Je la formulerai ainsi: pour comprendre plus exactement le rôle socialisant (dans le sens de 
gesellschaftsbildend) de la culture (ou des cultures) et pour pouvoir en juger de façon critique, il faut repenser la notion de culture. "If the making of a new society is our goal, then one way to begin is by making new categories." (Ryan, p.25)

L'orientation de principe devrait être aujourd'hui, à mon avis, la prise en considération d'un a priori technologique comme facteur, comme élément, qui traverserait toute culture, c'est-à-dire la mise en garde en face de tout rousseauisme (ou néo-rousseauisme) qui est à l'origine des oppositions et aliénations entre Culture et Civilisation, Savoir et Moralité, Culture artistique et Culture technique, Culture d'élite et Culture de masse, et ainsi de suite jusqu'à la mobilisation chauviniste d'une opposition (voire d'une inimitié) entre Culture allemande et Civilisation occidentale pendant la Première guerre mondiale, reprise par l'idéologie des Nazis.

On a dit que l'ordinateur crée un nouveau paradigme de la culture et de son histoire!

Dans le domaine de l'histoire des sciences, ce rousseauisme a été confirmé par la construction de cultures divergentes et contradictoires dont nous connaissons la problématique depuis les débats qu'a déchaînés le livre de Charles Percy Snow. La notion d'<<industrie culturelle $>>$ (Kulturindustrie ) établie par les auteurs de la Dialektik der Aufklärung dans les années 1940 et celle de Bewusstseinsindustrie de Hans Magnus Enzensberger (plus tard dans les années 1960) ne sont que la conclusion extrême de ce rousseauisme. Aujourd'hui l'élitisme de cette notion exclusive et excluante de la culture est évident. Ce qui est moins évident, ce sont les voies par lesquelles on en sortirait et les concepts théoriques qui permettraient de construire et d'analyser les nouveaux champs d'action culturelle.

Il est donc largement reconnu aujourd'hui que la notion de culture doit être constituée à partir du pluriel. Lynn Hart vient de nous rappeler à ce sujet que c'est chez l'anthropologue allemand Franz Boas, qui a fondé l'anthropologie culturelle proprement dite, qu'apparaît pour la première fois à la fin du siècle dernier la culture au pluriel. Dans un mémorandum sur l'état des sciences humaines dans notre culture contemporaine qui vient de paraître en Allemagne, les auteurs, de leur côté, distinguent une double signification à la culture: la culture "comme somme (Inbegriff) de tout travail humain et de toute forme de vie" et la culture comme "système particulier de cette culture générale qui se différencie par des fonctions différentes en culture technique, culture économique, culture politique, etc, ce qui serait un indice de l'inter-relation entre les deux sphères de culture, celle générale et celle particulière". (p.40)

Pour ne pas tomber dans le pur éclectisme (et dans un pan-culturalisme comme envers de la médaille) et afin de se donner des instruments théoriques pour comprendre les changements de fond survenus dans la culture, on s'est posé depuis quelques années la question de savoir si l'on ne 
devrait pas avancer par des voies nouvelles. "The reality in question is no longer revealed by clearly delineated concepts but rather by images belonging to a 'logic of the blurred", écrit par exemple le philosophe berlinois Dietmar Kamper (After Modernism: Outlines of an \#sthetics of Posthistory, 1990, p.113). Cette voie, à travers ce que certains nomment aujourd'hui une "nouvelle pensée esthétique" qui actualise le concept kantien du "sensus communis æstheticus", se veut explicitement politique à partir d'un constat de suspicion:

The suspicion that the decisive productive forces of the human race have become destructive, that a kind of 'suicide' is impending, can no longer be erased. It is an era of the pale death, an era into which humans have meanwhile entered by constructing one history [ou une seule culture, K.B.]. That is why the work on differences was intensified, occasionally even to the point of self-contradiction. Only an aesthetics of posthistory could make one aware of the unprecedented scandal rooted in the foundations of civilization - that humanity, acting in the frameworks of the story of salvation and progress, wants its decline. To escape this will, bristling with weapons as it does, would be the task that remains. (D.Kamper, p.11)

J'en tire la conclusion que le devoir de repenser la notion de culture exigerait une présupposition: celle de commencer par changer notre propre "culture de penser" qui, de son côté, exigerait une politique de la recherche et de l'enseignement d'un type nouveau.

3. Sur cette voie s'érigent des obstacles et des réticences importants. On les a caractérisés et décrits par la notion de "culture de la normalité" (Normalitätskultur ). Il s'agit de dispositifs et de mécanismes hautement différenciés et efficaces qui consistent à réduire (et à récupérer) tous les conflits et toutes les contradictions de la vie sociale et culturelle à des "normes", à des "équilibres", à des "consensus". En outre, cette "culture de la normalité" fonctionne de plus en plus par symbolisation en traduisant et en transformant au moyen de symboles collectifs (Kollektivsymbole) les anormalités et les écarts dans le discours de la normalité, et propose comme allant de soi que tout est "normalisable". Pour ne donner qu'un exemple, quand on a commencé en Allemagne, après la chute du mur de Berlin, à mettre en route "le train de l'unification" (en voilà un symbole collectif!) et que l'on a commencé à envisager les perspectives d'une unification dans les domaines de la culture et de la science, on a tout de suite soumis ce processus aux critères de la "culture de normalité". Suivant une espèce de "perfectibility fallacy" et des critères d'efficacité régis par la concurrence dans les systèmes économiques de marché libre, on a fait valoir la norme des critères occidentaux (western standards ) qui a servi de qualification négative de la culture et de la science en RDA.

Un élément plutôt anecdotique survenu lors d'une rencontre récente entre sociologues allemands de l'est et de l'ouest révèle l'assimilation (et le jeu de répression) du discours de la normalité. Lors d'une controverse, les 
collègues ouest-allemands critiquaient leurs collègues de l'est en disant qu'ils devraient d'abord faire l'apprentissage d'une "culture de discussion" avant de pouvoir se permettre d'intervenir dans les débats sur des questions thématiques sérieuses (Sachfragen). Il est significatif à cet égard que la définition avancée par Habermas pour expliquer les changements survenus avec la "Wende" en RDA, celle de "révolution rattrapante" (Nachholende Revolution ), est tout à fait conforme avec ce discours de la normalité. Car la mobilisation des jugements de valeur dans la tradition humaniste et celle de l'Aufklärung vise l'oubli et l'élimination de tout ce qui s'en écarte, et qui pourrait servir comme germe d'une subjunctive culture.

\section{Karlheinz Barck}

Académie des Sciences

Berlin

Surface Page d'Acceuil/Home Page 